\title{
A new measurement of the Cosmic-Ray Electron Spectrum with Fermi-LAT
}

\author{
Francesca R. Spada ${ }^{1, a}$ on behalf of the Fermi-LAT Collaboration \\ ${ }^{1}$ Istituto Nazionale di Fisica Nucleare sez. di Pisa \\ Largo B. Pontecorvo, 56127 Pisa, Italy
}

\begin{abstract}
Cosmic-ray electrons provide a probe of the origin and propagation mechanisms of cosmic rays in local galactic environment.

The Large Area Telescope (LAT), on board the Fermi mission, is a pair-conversion space telescope for high-energy electromagnetic radiation, operating in low Earth orbit since June 2008. The LAT has collected the largest high-energy cosmic-ray electron sample to date, with more than $10 \mathrm{k}$ events above $1 \mathrm{TeV}$. The event selection and reconstruction capability exploits the performance of the recently improved event-level analysis developed by the Fermi-LAT Collaboration.

We present a new measurement of the inclusive spectrum of cosmic-ray electrons and positrons performed on seven years of data, in an energy range from $7 \mathrm{GeV}$ up to $2 \mathrm{TeV}$.
\end{abstract}

\section{Introduction}

While propagating throughout the Galaxy, high-energy electrons and positron (CRE) rapidly lose energy by inverse Compton scattering on the interstellar radiation field and by synchrotron emission on the Galactic magnetic field. As a consequence, the CRE diffusion distance is of the order of $100 \mathrm{pc}$ at $1 \mathrm{TeV}$, much shorter than the radial scale of the Galaxy.

The shape of the CRE spectrum from about $100 \mathrm{GeV}$ up to few TeV can provide evidence of the presence of local CRE sources. These can be of astrophysical, like Supernova Remnants (SNR) or Pulsar Wind Nebulae (PWN), or of exotic nature, as the elusive dark matter.

Lower-energy CRE are affected by energy-dependent diffusive losses, convection in the interstellar medium, and perhaps reacceleration by second-order Fermi processes during transport from their sources. Since all these processes can affect the CRE spectrum after its injection by the sources, the observed spectrum is sensitive to the environment, determining how CRE propagate through the Galaxy.

A recent measurement by AMS-02 [1] has shown that the CRE spectrum can be fit with a single power law up to $1 \mathrm{TeV}$, with an index of $-3.170 \pm 0.008$. Measurements by H.E.S.S. [2, 3] give an indication of a cutoff at $2 \mathrm{TeV}$. These results can be jointly interpreted with the presence of local CRE sources with a spectral cutoff at about this energy.

ae-mail: francesca.spada@ cern.ch 


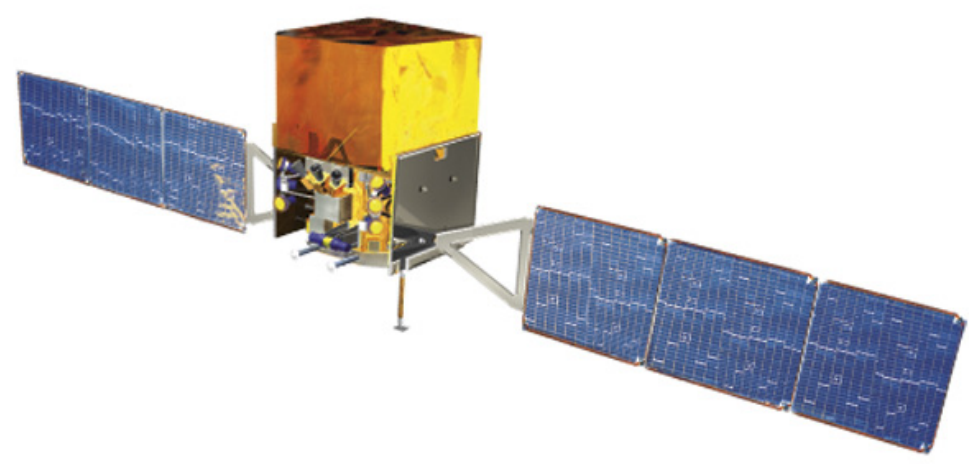

Figure 1: The Fermi satellite. The obsevatory carries two instruments on-board: the $\gamma$-ray Burst Monitor (GBM) [4] and the Large Area Telescope (LAT). The GBM, sensitive in the energy range between $8 \mathrm{keV}$ and $40 \mathrm{MeV}$, observes the full unocculted sky with rough directional capabilities - at the level of one to a few degrees - for the study of transient sources, in particular Gamma Ray Bursts (GRBs). The LAT is designed to detect $\gamma$-rays of energy greater than $20 \mathrm{MeV}$, where pair-conversion is the dominant interaction process, and is also naturally suitable to detect electrons and positrons.

\section{The Fermi mission}

The Fermi $\gamma$-ray Space Telescope is a NASA mission designed to survey the sky in the broad energy range from $20 \mathrm{MeV}$ to more than $300 \mathrm{GeV}$, with the additional capability of studying transient phenomena at lower energies and of detecting charged species, notably leptons, from $\mathrm{GeV}$ to $\mathrm{TeV}$ energies.

The Fermi satellite, shown in figure 1, was launched from Kennedy Space Center, Cape Canaveral, FL, on board a Delta II 7920-H rocket on July 11, 2008, and is in nominal science operation since August 4, 2008, orbiting at an altitude of $565 \mathrm{~km}$ with a $25.6^{\circ}$ inclination. The large field of view of the telescope $(2.5 \mathrm{sr})$ provides the ability to observe $20 \%$ of the sky at any time which, in the nominal scanning mode of operation, translates in a full sky survey every three hours.

Onboard Fermi, the Large Area Telescope (LAT) [5], exploiting state-of-the-art, high-energy physics detector techniques, is designed to detect $\gamma$-rays of energy greater than $20 \mathrm{MeV}$, where pairconversion is the dominant interaction process.

\subsection{The LAT instrument}

The LAT is a $4 \times 4$ array of identical towers, each made by a tracker-converter (TRK) and a calorimeter module (CAL). A segmented anti-coincidence detector (ACD) covers the tracker array, and a programmable trigger and data acquisition system completes the instrument.

Each TRK module features 16 tungsten layers, inducing the conversion of $\gamma$-rays into $\mathrm{e}^{+} / \mathrm{e}^{-}$pairs, and $18 x-y$ pairs of single-sided silicon strip detector planes, for a total of 1.5 radiation lengths of material on-axis. The silicon sensor technology allows precise tracking with no detector-induced dead time and no use of consumables, and the capability to self-trigger.

The calorimeter, described in more detail in section 2.2, provides an intrinsically threedimensional image of the shower development, which is crucial for both the energy reconstruction, 


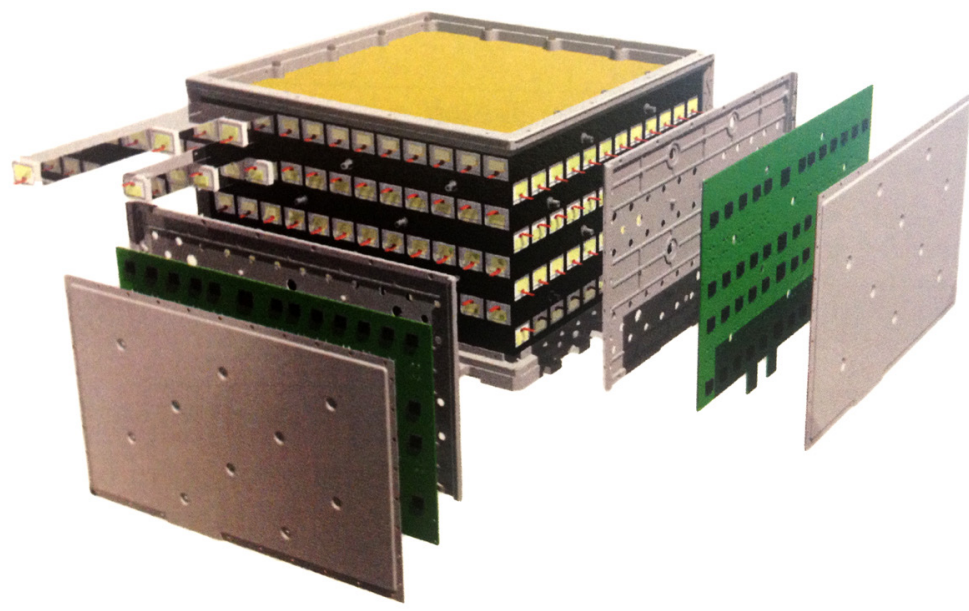

Figure 2: Exploded view of a calorimeter module with the hodoscopic $12 \times 8$ array of CsI blocks in the carbon fibre structure. Also shown are the readout electronics and the aluminum side panels providing shield against electromagnetic interference.

especially at high-energy where a significant part of the shower can leak out of the back of the instrument, and for background rejection.

The ACD, a set of plastic scintillators surrounding the tracker, serves as a veto against the overwhelming background due to charged cosmic rays. In order to limit the "self-veto" effect due to the backsplash of secondaries from high-energy particles hitting the calorimeter, it is segmented in 89 tiles providing spatial information that can be correlated with the signal from the tracker and the calorimeter. The overall power budget of the LAT is $650 \mathrm{~W}$.

Since electrons and positrons, as well as photons, develop electromagnetic showers in the CAL, and are detected in the TRK silicon modules, the LAT is naturally suitable to measure CRE direction and energy, up to the $\mathrm{TeV}$ scale. An on-board filter reduces the data rate to be downliked to Earth from about $10 \mathrm{kHz}$ to $300-500 \mathrm{~Hz}$. The so called gamma filter is specifically designed to reject charged particles, but it accepts all events with a raw deposited energy in the CAL greater than $20 \mathrm{GeV}$, so it does not prevent CRE to be accepted in the data sample. However, extending the spectrum measurement below this energy requires the use of an unbiased sample of all trigger types, that actually exists and is prescaled on-board by a factor 250. A dedicated analysis is needed, described in detail in section 3.3, and a unified approach is used wherever possible, with ad-hoc strategies when necessary.

\subsection{The electromagnetic calorimeter}

Figure 2 shows an exploded view of a calorimeter module, consistsing of $96 \mathrm{CsI}(\mathrm{Tl})$ scintillation crystals with PIN photodiode readouts, arranged in a hodoscopic configuration for a total depth of $\sim 8.6$ radiation lengths on axis. The eight layers contained in a module, each formed of 12 crystals, are rotated by $90^{\circ}$ with respect to the preceding and the following one, thus forming an $x-y$ array.

Each of the 1526 crystals forming the CAL provides a measurement of both the amount of energy released, and the three-dimensional location of the energy deposition, thus allowing the imaging of 

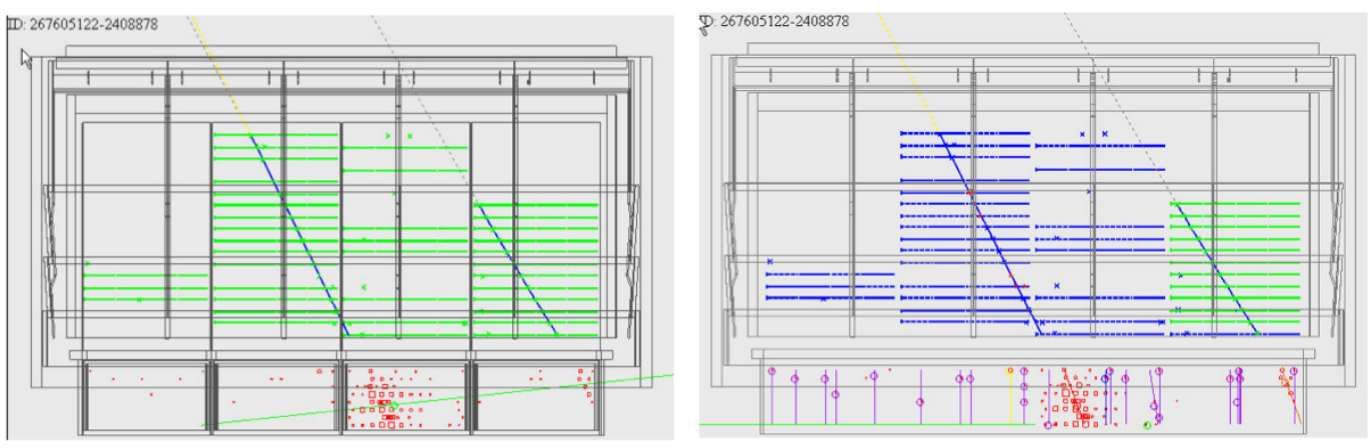

Figure 3: Effect of the CAL clustering stage on the event reconstruction, in a typical event in which ghost activity appears alongside with the signal produced by a real gamma-ray crossing the detector. Left panel: a single particle in the LAT is assumed, resulting in a flipped shower axis (green line at the bottom). Right panel: clustering the activity in the CAL, the gamma-ray is isolated and the shower axis correctly reconstructed (red line).

the shower development. Two dual-photodiode assemblies, measuring the scintillation light produced in the crystal and containing a large-area photodiode for small energy depositions, and a small-area photodiode to measure large energy releases, are located at each end. The readout is performed by a custom analog amplifier ASIC, that minimized the power consumption and allows to cover the large dynamic range of the input signal.

Nonetheless, extending the CRE energy measurement beyond $1 \mathrm{TeV}$ with the LAT is challenging because, at such high energy, only $35 \%$ of the shower is typically contained in the CAL and a significant fraction of the crystals along the shower axis are saturated, since crystal saturation occurs for CRE above $600 \mathrm{GeV}$. This makes both the energy reconstruction and the hadronic background rejection extremely difficult.

\subsection{Pass 8 event-level analysis}

The event selection developed for the LAT before launch has been periodically updated over the years to reflect the constantly improving knowledge of the detector and the environment in which it operates. Pass 8, a radical update of the entire event-level analysis released to the public in 2015, represents the most recent iteration [6]. The revision involved every aspect of the data processing, from the simulation of the detector to the event reconstruction and the background rejection. The improvements include (but are not limited to) a significant reduction in background contamination, an increased effective area, a better point-spread function, a better understanding of the systematic uncertainties and an extension of the energy reach for the photon analysis below $100 \mathrm{MeV}$ and above a few hundred $\mathrm{GeV}$. The calorimeter reconstruction has been extensively revisited at all levels - the crystal simulation, the energy and direction reconstruction, the background-rejection algorithms.

Extremely relevant to the CRE spectrum measurement is the fact that in pass 8 a clustering stage in the calorimeter was introduced, aimed at identifying and isolating residual ghost signals away from the gamma-ray shower, that can introduce substantial errors in the measurement of the energy, centroid and direction of the shower. This allowed to recover the loss in effective area coming from events misclassified as background, due to the fact that the matching between the tracker and the 


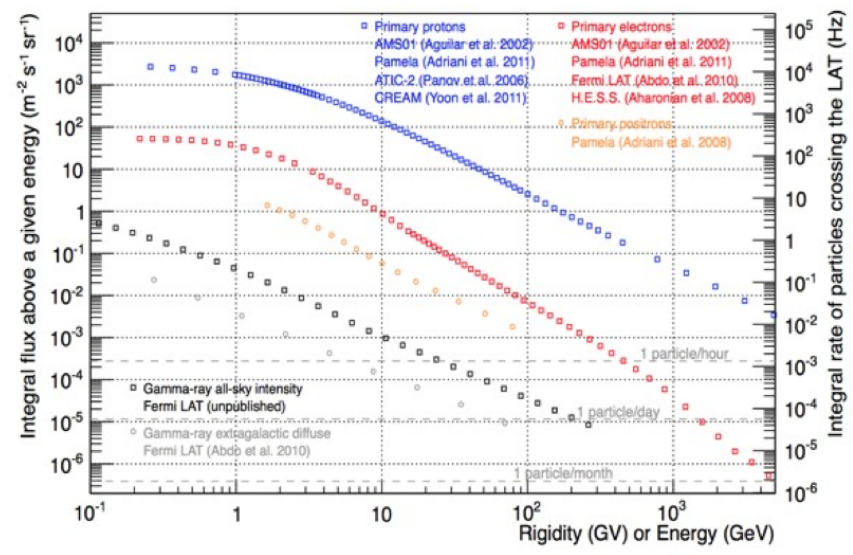

Figure 4: Integral fluxes for a few of the most abundant particle species at low-Earth orbit. Blue: primary protons, that represent the most important background source for this analysis; red: primary electrons and yellow: primary positrons, i.e. (summed) the signal.

calorimeter is one of the main inputs to the background rejection. Figure 3 shows the effect of the CAL clustering stage on the event reconstruction: the signal produced by a gamma-ray crossing the detector is decoupled from the ghost activity far from the real particle.

Above a few $\mathrm{GeV}$, the particle energy is determined from a three-dimensional profile fit to the calorimeter layer energies. This approach is optimal up to $1 \mathrm{TeV}$, where the average energy release per crystal at the shower maximum starts exceeding the dynamic range of the readout electronics and saturation becomes an issue, as anticipated in section 2.2. In order to overcome this limitation, the profile fit has been extensively reworked taking into account individual crystal energies at each layer. Concerning the contributions from saturated channels, Monte Carlo simulations indicate that their energy deposits can be recovered to some extent by using the information from the nearby (nonsaturated) crystals, thus extending the energy measurement range up to $\sim 3 \mathrm{TeV}$.

\section{The analysis}

Figure 4 shows the integral fluxes for a few particle species in cosmic rays at low-Earth orbit. It is evident that to extract our signal, consisting of electrons and positrons reaching the detector from all directions, we must be able to get rid of a much more abundant background, mainly due to protons, since $\mathrm{n}_{\mathrm{p}} / \mathrm{n}_{\mathrm{e}} \sim 500$ for $\mathrm{E}>100 \mathrm{GeV}$.

Thanks to the new pass 8 event analysis, with its improved algorithms for track and shower reconstruction, as well as powerful multivariate methods for background suppression, we can achieve a level of background contamination smaller than $25 \%$ and an energy resolution better than $20 \%$ up to $2 \mathrm{TeV}$.

The LAT on-board gamma filter is designed to reject charged particles, but it accepts all events with a deposited energy in the CAL larger than $20 \mathrm{GeV}$, thus preserving high-energy charged particles from being lost from the data sample. However, extending this analysis below $20 \mathrm{GeV}$ requires the use of the unbiased sample of all trigger types, that is prescaled on-board by a factor 250 .

As we did in the previous LAT measurement of the CRE spectrum [7], we performed two independent analyses: a High-Energy (HE) analysis above $42 \mathrm{GeV}$, and a Low-Energy (LE) analysis between 


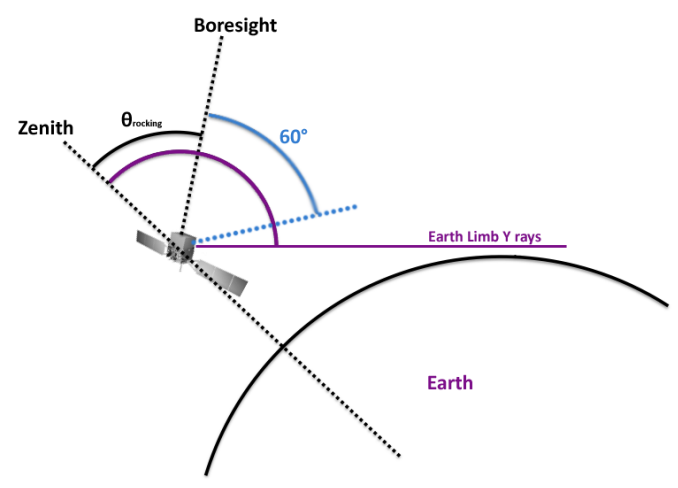

Figure 5: At the average Fermi orbital heigth, Earth limb photons reach the detector at a $113^{\circ}$ angle from the Earth zenith direction. In standard survey mode, i.e. with a LAT rocking angle of $51^{\circ}$, getting rid of the background due to limb photons requires rejecting particles coming at an angle greater than $60^{\circ}$ from the boresight direction.

7 and $70 \mathrm{GeV}$. We chose to use a unified approach wherever possible, with ad-hoc strategies when needed. Both analyses, however, require a high-quality starting sample, a first background rejection step, and a final selection to reduce as much as possible the residual proton contamination.

\subsection{Event selection}

The starting sample consists of LAT pass 8 data collected in standard survey mode, i.e. when the rocking angle of the LAT from the zenith is less than $51^{\circ}$, for an overall live time of 4.68 years.

Earth limb photons appear as a sharp peak located at $113^{\circ}$ from the Earth zenith direction. The rocking angle selection, with an additional one on events coming within $60^{\circ}$ from the LAT boresight, eliminate a potential contamination from this source (see figure 5).

Quality cuts consist in requiring events with a well reconstructed track, and whose path length through the CAL is larger than 8 radiation lengths. Additionally, events with a $\chi^{2} /$ n.d.f. $>60$ for the shower profile fit are rejected.

In order to remove $\alpha$ particles and heavier ions, we add requirements on the path-length corrected signal in the ACD and on the TKR time over threshold: both variables are in fact sensitive to the ionization signal, that is proportional to $Z^{2}$, thus providing charge-deposition information. Applying a cut in the plane spanned by these two variables around the region corresponding to $Z=1$ reduces the contamination to less than a few per mill with respect to protons. The residual contribution from celestial photons also is reduced to less than $1 \%$ of the CRE flux.

For the LE analysis, an additional selection is applied that requires more than $2 \mathrm{GeV}$ deposited in the CAL and depends on the geomagnetic cutoff rigidity.

We are now left with a proton background only, that requires a dedicated strategy. The chosen approach is based on the multivariate analysis toolkit TMVA [8] making use of boosted decision trees (BDT), that were trained on Monte Carlo-simulated datasets of electrons and positrons (the signal) and protons (the background).

To account for rapid changes with the energy of the typical event topology in the detector, we define 8 energy bins in $\log _{10}(\mathrm{E} / \mathrm{GeV}$ ) equally spaced between 1.5 ad 3.5 (corresponding to $31.6 \mathrm{GeV}$ 

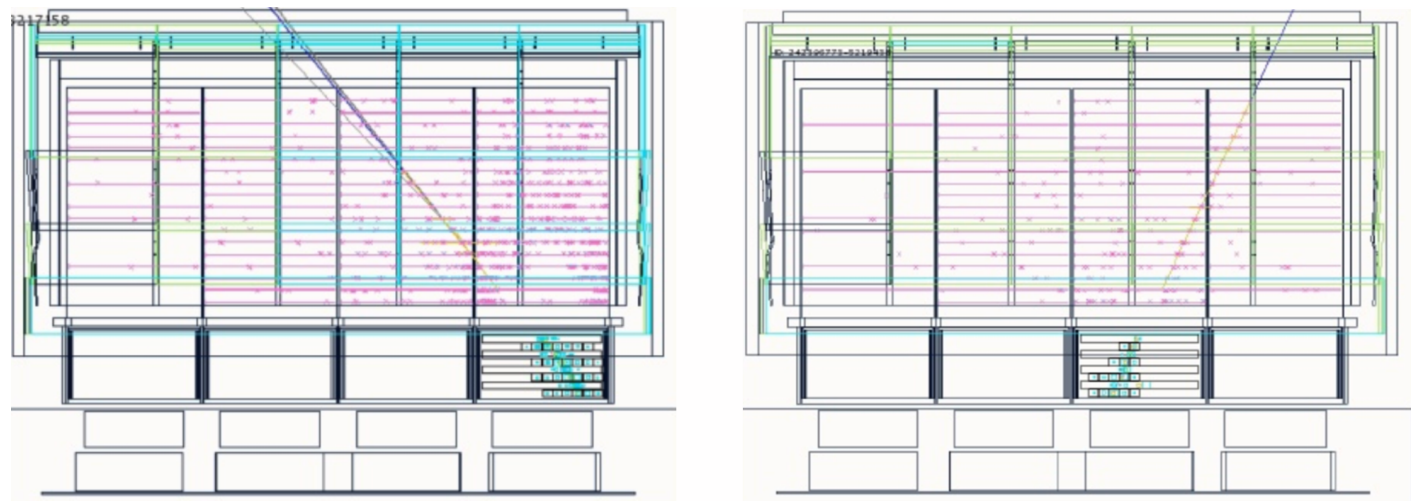

Figure 6: Different incident particles determine different event topologies in the LAT. Left: 1 TeV proton (background). Right: $1 \mathrm{TeV}$ electron (signal).

and 3.16 TeV) for the HE, plus 1 energy bin for the LE, and separately train a BDT in each bin. All BDTs are built from the same 19 observables, chosen according to good Monte Carlo-data agreement and high separation efficiency, that are related to the event topology in TKR and CAL. Five concern the position of the shower in the detector, while the others describe the shower topology in the TKR and $\mathrm{CAL}-$ as e.g. the shower transverse size that, as evident from figure 6 , looks quite different in the case of signal (right) and background (left), thus providing a high discriminating power.

\subsection{High energy}

The validity of the BDT approach strongly relies on the assumption that Monte Carlo well reproduces the distributions of the variables in the data. For a few of the original Monte Carlo outputs this was not the case above a few hundreds of $\mathrm{GeV}$, and this discrepancy had a significant impact on the effectiveness of the BDT method. In order to mitigate these discrepancies, we derived additive individual
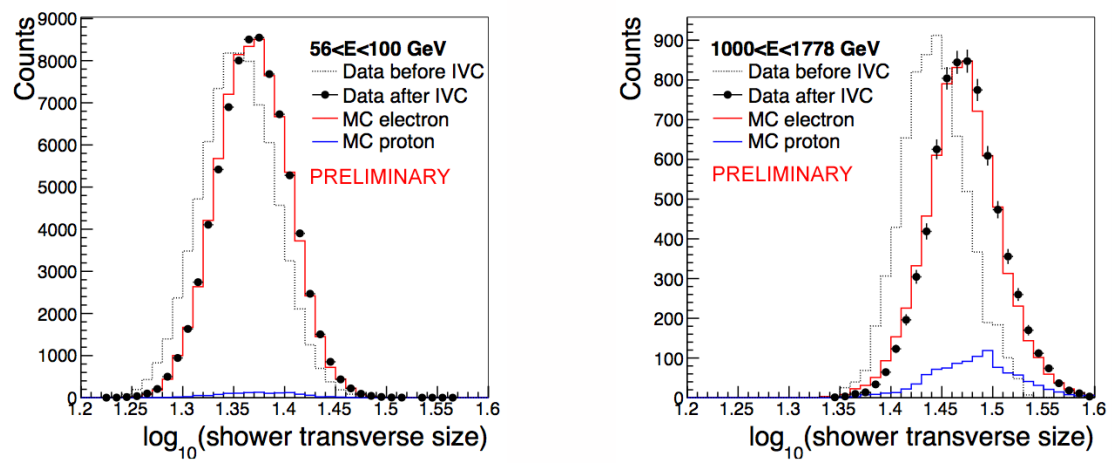

Figure 7: The shower transverse size before (dotted lines) and after (black circles) IVC correction for events between 56 and $100 \mathrm{GeV}$ (left) and between 1 and $1.78 \mathrm{TeV}$ (right). The red histograms correspond to the electron simulation, and the blue ones to the residual background. 

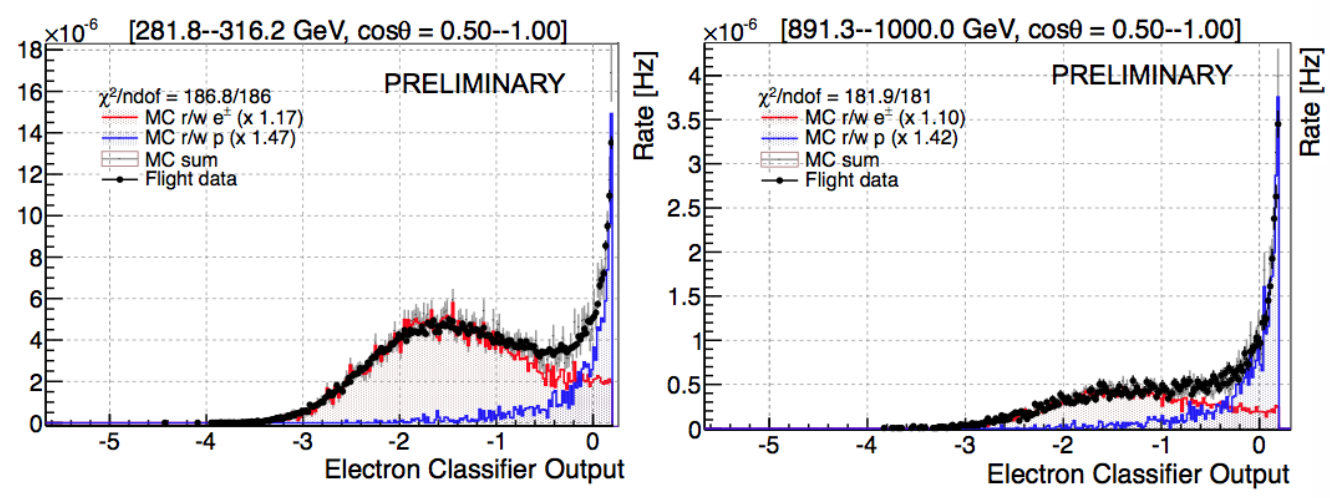

Figure 8: Template fit results for the HE analysis in two different energy bins. The Monte Carlo prediction (grey line) is the sum of the signal (red) and background (blue) templates, scaled by the normalization factors given by the template fit and reported in brackets in the caption. The thus rescaled simulation well reproduces the data distribution (black dots).

variable corrections (IVC) for the data as a function of energy and incidence angle of the particle. The calibration is applied to the data, which is equivalent to applying it to the simulation, but has the advantage that there is no need to retrain the BDTs. The IVCs provide a significant improvement in the agreement for all the variables used as input to the BDTs, and the residual differences are taken into account in the estimate the systematic uncertainties. Figure 7 shows, as an example, the IVC impact on the data-simulation agreement for the shower transverse size in two different energy bins.

Each BDT provides an output variable $p_{B D T}$ assuming values between -1 (candidate protons) and 1 (candidate electrons), whose distribution for electrons peaks very sharply at 1 . It is then convenient to build an estimator as

$$
P_{C R E}=\log _{10}\left(1-p_{B D T}\right)
$$

For each energy bin, we then construct the Monte Carlo electron and proton distributions of $P_{C R E}$ and use them as templates. The sum of the two templates is fit to the data by varying their normalizations, and the renormalized proton template is used to estimate the number of background events in the data integrating the $P_{C R E}$ distribution up to a chosen cut value. The so obtained number of background events is subtracted to the number of events in the data to obtain the number of CRE events. The cut on $P_{C R E}$ is energy-dependent and is such that the efficiency for electrons goes from $90 \%$ at $30 \mathrm{GeV}$ to $80 \%$ at $300 \mathrm{GeV}$, and then down to $50 \%$ at $2 \mathrm{TeV}$ and above. This choice is driven by the increasing difficulty of the background rejection with energy, particularly above $300 \mathrm{GeV}$. The analysis stops when the residual contamination reaches $20 \%$, which occurs at $2 \mathrm{TeV}$. One example of the result of the template fit, for two different energy bins, is shown in figure 8, where the Monte Carlo prediction is the sum of the signal and background templates scaled by the normalization factors given by the template fit to the data, and clearly well reproduces the data distribution.

\subsection{Low energy}

Due to the on-board gamma filter, extending the analysis below $20 \mathrm{GeV}$ requires the use of the unbiased sample of all trigger types, that is prescaled by a factor 250 . The LE selection is based on the 


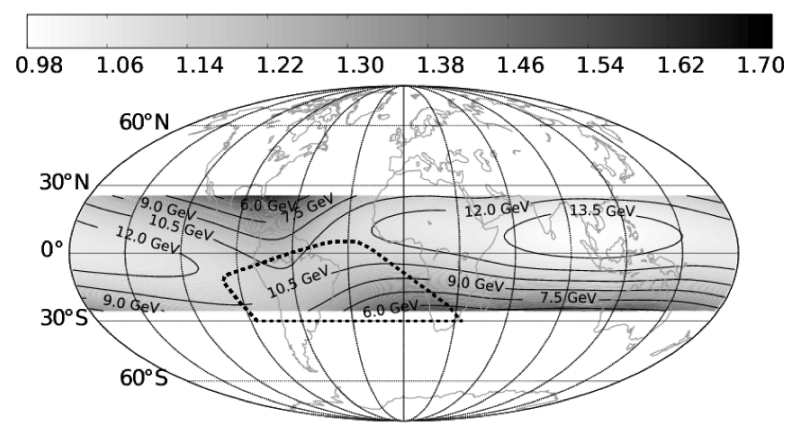

Figure 9: Map of the Earth showing Mcllwain- $L$ values (greyscale) in the region spanned by the LAT orbit. Higher McIlwain- $L$ values (i.e., darker regions) correspond to lower rigidity cutoff values (contour lines).

same multivariate analysis approach as used for the HE selection, and a dedicated classification tree is trained in the low-energy region. Contrary to the HE case, the background Monte Carlo sample used for the training is a realistic simulation of cosmic rays in low Earth orbit and of Earth limb photons [9]. Since the LE analys stops at $70 \mathrm{GeV}$, no IVC correction, relevant at high energy, is applied to the data. The residual background contamination is estimated by the same template fitting technique used in the $\mathrm{HE}$ analysis.

Below $\sim 20 \mathrm{GeV}$, the CRE flux reaching the LAT is strongly influenced by the shielding effect of the Earth magnetic field: at any given geomagnetic position, Galactic charged particles can reach the detector only if they are above a certain rigidity $R_{C}$. The dependence of this rigidity cutoff on the coordinates can be conveniently parametrized by the McIlwain- $L$ parameter [10], that describes the set of geomagnetic field lines crossing the magnetic equator at a distance of $L$ Earth radii. Geographic coordinates that are magnetically equivalent from the standpoint of incoming charged particles thus share the same McIlwain- $L$, which is then an optimal parameter for describing cutoff rigidities. Measuring the CRE spectrum at a given energy $E$ requires selecting data collected in a McIlwain- $L$ region in which $R_{C}<E$.

A map of the Earth in McIlwain- $L$ is shown in figure 9. The LAT orbit spans an interval $L=\{0.98-1.73\}$, corresponding to vertical rigidity cutoff values from $\sim 6 \mathrm{GeV}$ to $\sim 14 \mathrm{GeV}$. The $L$ range is thus divided in 15 bins, and the corresponding $R_{C}$ values are determined from a fit of the count spectrum. This provides an empirical relation $R_{C}=f(L)$, that can then be used to find, for each energy bin of the CRE spectrum, the McIlwain- $L$ value $L_{\min }$ corresponding to the lower boundary of the energy bin itself, and select data collected in regions with $L>L_{\min }$.

However, the cutoff is smooth, and even above the nominal $R_{C}$ there is still a non-negligible fraction of electrons and positrons which are prevented from reaching the detector by the magnetic shadow of the Earth. The fraction of undetected CRE is estimated with the help of the particle trajectory tracing code developed by Smart and Shea [10] and the 2010 model of the Earth's magnetic field from the International Geomagnetic Reference Field (IGRF) [11]. The tracer computes the trajectory of test particles - generated according to a power law with an index of -3.2 using the abundance ratios measured by AMS-02 for electrons and positrons [12] and passing the standard analysis event selection - starting from the spacecraft, that is each time assigned a realistic orbital position with a probability based on real data. Test particles with a trajectory reaching 20 Earth radii correspond to CRE that could actually be observed by the instrument, while trajectories intersecting the Earth's atmosphere 


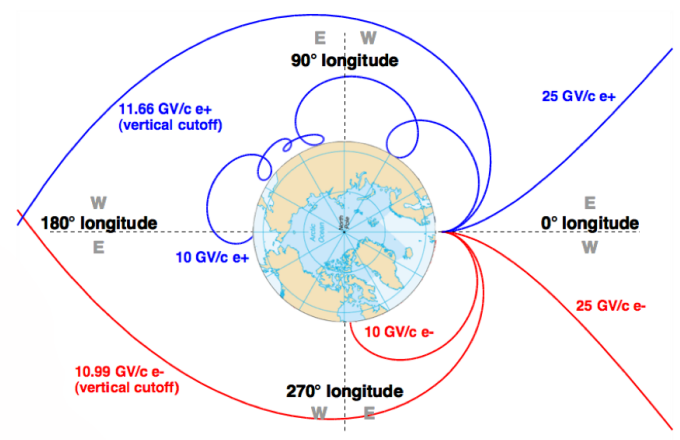

Figure 10: Trajectories of test particles in the Earth magnetic field computed by the tracing code. Red lines represent electrons, blue ones represent positrons. At a given orbital position, particles behave depending on their energy. Test particles with a trajectory reaching 20 Earth radii correspond to CRE that could actually be observed by the instrument, while trajectories intersecting the Earth's atmosphere correspond to lost particles.

correspond to lost particles, as explained in figure 10. The observed flux is then corrected accordingly, with the derived correction factor increasing from $+10 \%$ at $7 \mathrm{GeV}$ to $+40 \%$ at $15 \mathrm{GeV}$, decreasing down to $+5 \%$ at $30 \mathrm{GeV}$.

\subsection{Systematics}

The control of the absolute energy scale is key to the measurement of the CRE spectrum. A selfconsistent check comes from a comparison of the CRE cutoff measured in LAT data with the one predicted by the tracer as described in section 3.3. We find an average ratio $R_{C}^{\text {data }} / R_{C}^{\text {tracer }}=1.033 \pm$ 0.004 (stat) \pm 0.020 (syst). The calibration of the CAL CsI crystals ensures a linearity better than $1 \%$ up to the saturation level. Assuming that the light yield is linear for electron showers up to $2 \mathrm{TeV}$, we rescale the event energies in data by $-3.3 \%$ in both the LE and HE analyses (here the IVC correction is applied afterwards). The in-flight absolute energy scale measurement gives a systematic uncertainty on the energy scale at $10 \mathrm{GeV}$ of $2 \%$. We estimate the uncertainty at $1 \mathrm{TeV}$ to be $6 \%$.

For the HE analysis, we considered three additional sources of systematic uncertainty, as shown in figure 11:

\section{A Acceptance}

The main uncertainty is due to the estimation of the effective area. We vary the cut on $P_{C R E}$ in a range corresponding to $\pm 20 \%$ in signal efficiency, obtaining a flux variation of $\sim 2 \%$ up to $1 \mathrm{TeV}$, increasing to $9 \%$ at $2 \mathrm{TeV}$;

\section{B IVC correction}

We move the correction factors, derived from the difference between the peak position of the data and Monte Carlo distributions for each BDT input variable, by its $1-\sigma$ uncertainty, getting a signal variation from $2 \%$ at $40 \mathrm{GeV}$ to $12 \%$ at $2 \mathrm{TeV}$;

\section{Residual fraction of background events}

To take into account the uncertainty on the GEANT4 prediction of the fraction of protons mim- 

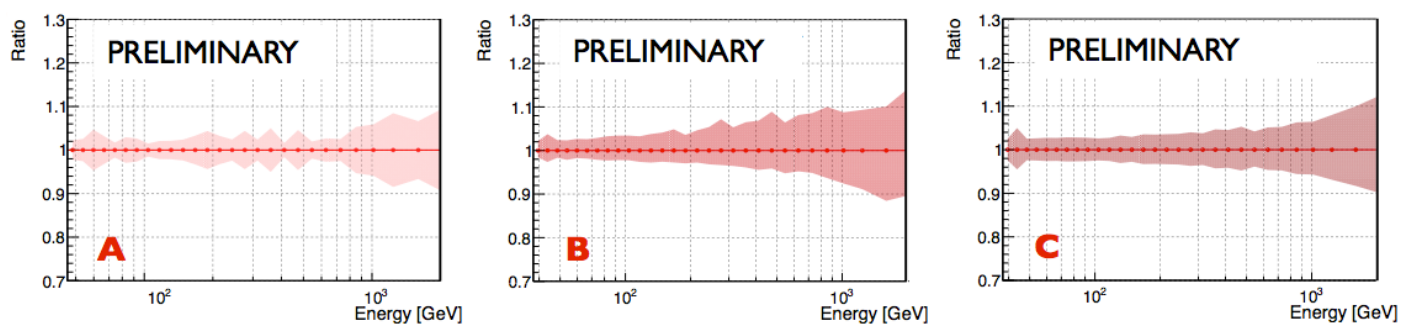

Figure 11: Main sources of systematic error in the CRE measurement, represented with energy as a band including the ratio of the values obtained by varying analysis parameters with the central ones.

icking electromagnetic showers, we assume a $20 \%$ uncertainty on the number of background events, getting a signal variation $<2 \%$ up to $1 \mathrm{TeV}$ that increases to $6 \%$ at $2 \mathrm{TeV}$.

\section{Conclusions}

The CRE inclusive spectrum is shown in figure 12. The shaded band represents the systematics and does not include the one on the energy scale. Between $50 \mathrm{GeV}$ and $2 \mathrm{TeV}$, the spectrum is compatible with a single power law with a spectral index $-3.07 \pm 0.02$. Below $100 \mathrm{GeV}$, the new LAT measurement differs from the previous one [7] by 10-30\%. A large part of this difference is due to the fact that, in the previous analysis, the simulation that was used did not take into account the presence of ghosts described in section 2.3. Taking into account the uncertainties on the energy scale, this measurement is in agreement with the AMS and HESS ones.

\section{References}

[1] M. Aguilar et al. [AMS Collaboration], Phys. Rev. Lett. 113 (2014) 221102

[2] F. Aharonian et al. [H.E.S.S. Collaboration], Phys. Rev. Lett. 101 (2008) 261104

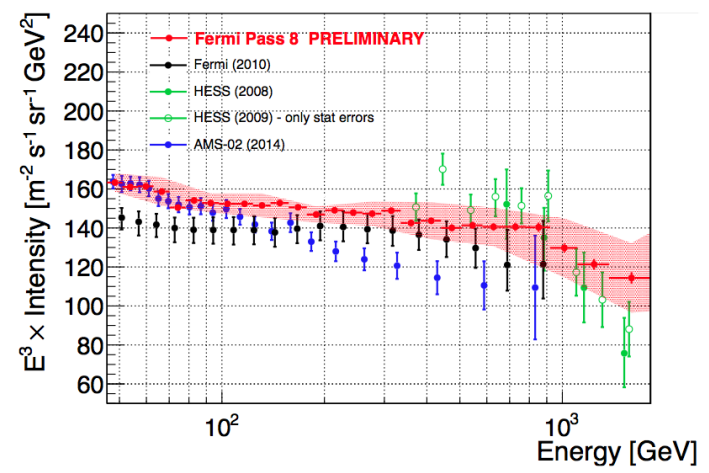

Figure 12: The CRE inclusive spectrum. The shaded band represents the systematics and does not include the one on the energy scale. 
[3] F. Aharonian et al. [H.E.S.S. Collaboration], Astron. Astrophys. 508 (2009) 561

[4] C. Meegan et al., AIP Conf. Proc. 921 (2007) 13

[5] W. B. Atwood et al. [Fermi-LAT Collaboration], Astrophys. J. 6971071 (2009)

[6] W. Atwood et al. [Fermi-LAT Collaboration], arXiv:1303.3514 [astro-ph.IM]

[7] M. Ackermann et al. [Fermi-LAT Collaboration], Phys. Rev. D 82 (2010) 092004

[8] A. Hocker et al., PoS ACAT (2007) 040 [physics/0703039 [PHYSICS]]

[9] T. Mizuno, T. Kamae, G. Godfrey, T. Handa, D. J. Thompson, D. Lauben, Y. Fukazawa and M. Ozaki, Astrophys. J. 614 (2004) 1113

[10] D. F. Smart and M. A. Shea, Advances in Space Research 36 (2005) 2012

[11] C. C. Finlay et al., Geophys. J. Int. 183 (2010) 12161230

[12] L. Accardo et al. [AMS Collaboration], Phys. Rev. Lett. 113 (2014) 121101 